\title{
Estimated number of tuberculosis in cases of municipality Capanema/PA for the year 2015 using the Method Sized Mobile
}

\section{Camily Murrieta Vasconcelos Oliveira Bezerra ${ }^{1}$, José Geraldo Gonçalves da Silva², Clauderino da Silva Batista ${ }^{3}$}

\author{
1,2Universidade Federal do Pará - UFPA. Campus Castanhal - Av. dos Universitários, Jaderlândia, Castanhal, PA. CEP: 68746-360. \\ ${ }^{3}$ Universidade Federal do Pará - UFPA. Campus Guamá - Rua Augusto Correa No 01. Belém, PA. CEP:66075-110. \\ Email:camilymv@hotmail.com,jg@ufpa.br, clauderino@ufpa.br
}

Received: August $24^{\text {th }}, 2016$

Accepted: September $17^{\text {th }}, 2016$

Published: December 22 ${ }^{\text {th }}, 2016$

Copyright $(2016$ by authors and Institute of Technology Galileo of Amazon (ITEGAM) This work is licensed under the Creative

Commons Attribution International

License (CC BY 4.0).

http://creativecommons.org/licenses/by/4.0/ (c) (1) (3) Open Actes:

\begin{abstract}
A decade ago, the World Health Organization (WHO) declared tuberculosis (TB) in a state of emergency in the world, which is still the leading cause of death from infectious disease in adults. According to WHO estimates, two billion people representing a third of the world population is infected with Mycobacterium tuberculosis. Of these, 8 million develop the disease and two million die each year. Tuberculosis is still a serious public health problem recognized by the Brazilian government. Therefore, due to their public policy purposes, assumed commitments with their citizens and the international community to control its evolution, seeking to reduce its prevalence in the population. In the Amazon, where about $60 \%$ of the country's indigenous population, the average incidence of tuberculosis has reached 75.1 cases per 100,000 population in 1980-2000, the highest among Brazilian geopolitical regions (the national average for this period was 59.2). In order to statistically verify the number of TB cases that may occur in the municipality of Capanema / PA based on the number of cases of the disease in previous years, there was a correlation capability of the Moving Average method for data collected as method useful to estimate the number of cases of this disease in 2015.
\end{abstract}

Keywords: Tuberculosis, the Moving Average Method, World Health Organization.

\section{Estimativa do número de casos de tuberculose no Município de Capanema/PA para o ano de 2015 utilizando o Método das Médias Móveis}

\section{RESUMO}

Há uma década, a Organização Mundial da Saúde (OMS) declarou a tuberculose (TB) em estado de emergência no mundo, onde ainda é a maior causa de morte por doença infecciosa em adultos. Segundo estimativas da OMS, dois bilhões de pessoas correspondendo a um terço da população mundial está infectada pelo Mycobacterium tuberculosis. Destes, 8 milhões desenvolverão a doença e 2 milhões morrerão a cada ano. A tuberculose ainda é um sério problema da saúde pública reconhecido pelo governo brasileiro. Portanto, em razão de propósitos de suas políticas públicas, assumiu compromissos com seus cidadãos e com a comunidade internacional de controlar sua evolução, procurando reduzir sua prevalência na população. Na Amazônia, onde vive cerca de $60 \%$ da população indígena do país, a incidência média da tuberculose atingiu 75,1 casos por 100.000 habitantes no período 1980-2000, a mais elevada dentre as regiões geopolíticas brasileiras (a média nacional para esse período foi de 59,2). Com o intuito de verificar estatisticamente a quantidade de casos de tuberculose que podem ocorrer no município de Capanema/PA baseado no número de casos da doença nos anos anteriores, verificou-se a capacidade de correlação do Método das Médias Móveis para os dados coletados como método útil à estimativa do número de casos desta doença no ano de 2015.

Palavras Chaves: Tuberculose, Método das Médias Móveis, Organização Mundial da Saúde. 


\section{INTRODUÇÃO}

Tuberculose é uma doença infectocontagiosa causada por uma bactéria que afeta principalmente os pulmões, mas também pode ocorrer em outros órgãos do corpo, como ossos rins e meninges.

Há uma década, a [1] declarou a tuberculose (TB) em estado de emergência no mundo, onde ainda é a maior causa de morte por doença infecciosa em adultos. Segundo estimativas da [1], dois bilhões de pessoas correspondendo a um terço da população mundial está infectada pelo Mycobacterium tuberculosis. Destes, 8 milhões desenvolverão a doença e 2 milhões morrerão a cada ano. [2] que cerca de $95 \%$ dos casos de Tb ocorrem no terceiro mundo, e, aí ocorrem $98 \%$ dos óbitos.

Em 22 países concentram-se $80 \%$ dos casos estimados para o mundo, entre os quais encontra-se o Brasil ocupando o $10 \%$ lugar (em 1997). O Brasil ocupa o $15^{\circ}$ lugar entre os 22 países responsáveis por $80 \%$ do total de casos de tuberculose no mundo. Estima-se uma prevalência de 50 milhões de infectados com cerca de 111.000 casos novos e 6.000 óbitos, ocorrendo anualmente.

Diante da atual situação, há necessidade de investimentos na qualificação dos serviços de saúde, na capacitação dos recursos humanos para as atividades de vigilância, avaliação e controle, de modo a ampliar a capacidade de diagnóstico por meio da baciloscopia, promover a cura, intensificar a busca do sintomático respiratório e dos contatos dos pacientes, nos municípios brasileiros e especialmente nos municípios prioritários para o Programa Nacional de Controle da Tuberculose.

A tuberculose ainda é um sério problema da saúde pública reconhecido pelo governo brasileiro. Portanto, em razão de propósitos de suas políticas públicas, assumiu compromissos com seus cidadãos e com a comunidade internacional de controlar sua evolução, procurando reduzir sua prevalência na população.

Na Amazônia, onde vive cerca de $60 \%$ da população indígena do país, a incidência média da tuberculose atingiu 75,1 casos por 100.000 habitantes no período 1980-2000, a mais elevada dentre as regiões geopolíticas brasileiras (a média nacional para esse período foi de 59,2) [3]. O impacto dessa endemia sobre as populações indígenas tem sido de grande magnitude, conforme apontam vários estudos realizados nas regiões amazônica e CentroOeste [4][5][6][7].

Análise recente evidenciou incidências de tuberculose de 286,8 e 326,8 casos por 100.000 habitantes para a população indígena na Amazônia, em 2000 e 2001, respectivamente [8]. Em Rondônia e regiões vizinhas, a tuberculose não apenas contribuiu para o significativo declínio populacional verificado em diversas etnias ao longo do século $\mathrm{XX}$, como também permanece como proeminente causa de morbimortalidade [9].
Neste contexto, a Matemática pode ser de grande utilidade ao possibilitar o cálculo de estimativas do número de casos da doença, a partir de dados coletados ao longo dos anos. Dentre os métodos de previsão disponíveis, o Método das Médias Móveis é muito utilizado, pois apresenta grande facilidade na manipulação dos dados coletados. Podemos sinteticamente classificar os métodos de previsão em: qualitativo e quantitativo, cujo método pode ser baseado em modelos matemáticos e utilizando dados diversos e demandas passadas para se chegar a uma previsão futura. Podem ser: métodos causais ou séries temporais.

\section{REVISÃO BIBLIOGRÁFICA}

\section{II.1 TUBERCULOSE}

\section{II.1.1 DEFINIÇÃO DE TUBERCULOSE}

A tuberculose - chamada antigamente de "peste cinzenta" [10], e conhecida também em português como tísica pulmonar [11] ou "doença do peito" - é uma das doenças infecciosas documentadas desde mais longa data e que continua a afligir a Humanidade nos dias atuais. É causada pelo Mycobacterium tuberculosis, também conhecido como bacilo de koch. Estima-se que a bactéria causadora tenha evoluído há 50.000 anos, a partir de outras bactérias do gênero Mycobacterium.

A tuberculose é considerada uma doença socialmente determinada, pois sua ocorrência está diretamente associada à forma como se organizam os processos de produção e de reprodução social, assim como à implementação de políticas de controle da doença. Os processos de produção e reprodução estão diretamente relacionados ao modo de viver e o trabalho do indivíduo. A tuberculose é uma doença que tem cura e o tratamento é viabilizado integralmente pelo SUS. Ainda assim, representa problema grave de saúde pública, com maior incidência entre populações vulneráveis, como: Indígenas, população vivendo com HIV/Aids, população privada de liberdade e moradores em situação, que tem a probabilidade de adoecer até 67 vezes [12] mais e que muitas vezes não conseguem acessar os serviços de saúde.

\section{II.1.2 TUBERCULOSE NO BRASIL E NO MUNDO}

Segundo a [1], a taxa de incidência da tuberculose vem diminuindo desde $2002 \mathrm{em} \mathrm{1,3 \%} \mathrm{ao} \mathrm{ano.} \mathrm{A} \mathrm{estimativa} \mathrm{de} \mathrm{novos}$ casos de tuberculose no mundo e de 8,8 milhões em 2010, equivalente a 128/100.000 habitantes. Desse total, 59\%, 26\%, 7\%, $5 \%$ e $3 \%$, respectivamente, estão na Ásia, África, região do Mediterrâneo, Europa e Américas (Figura 1). Entre 1 e 1,2 milhões de casos são de pessoas vivendo com HIV/AIDS. A África e responsável por $82 \%$ dos casos de coinfecção tuberculose/ HIV no mundo [1]. 

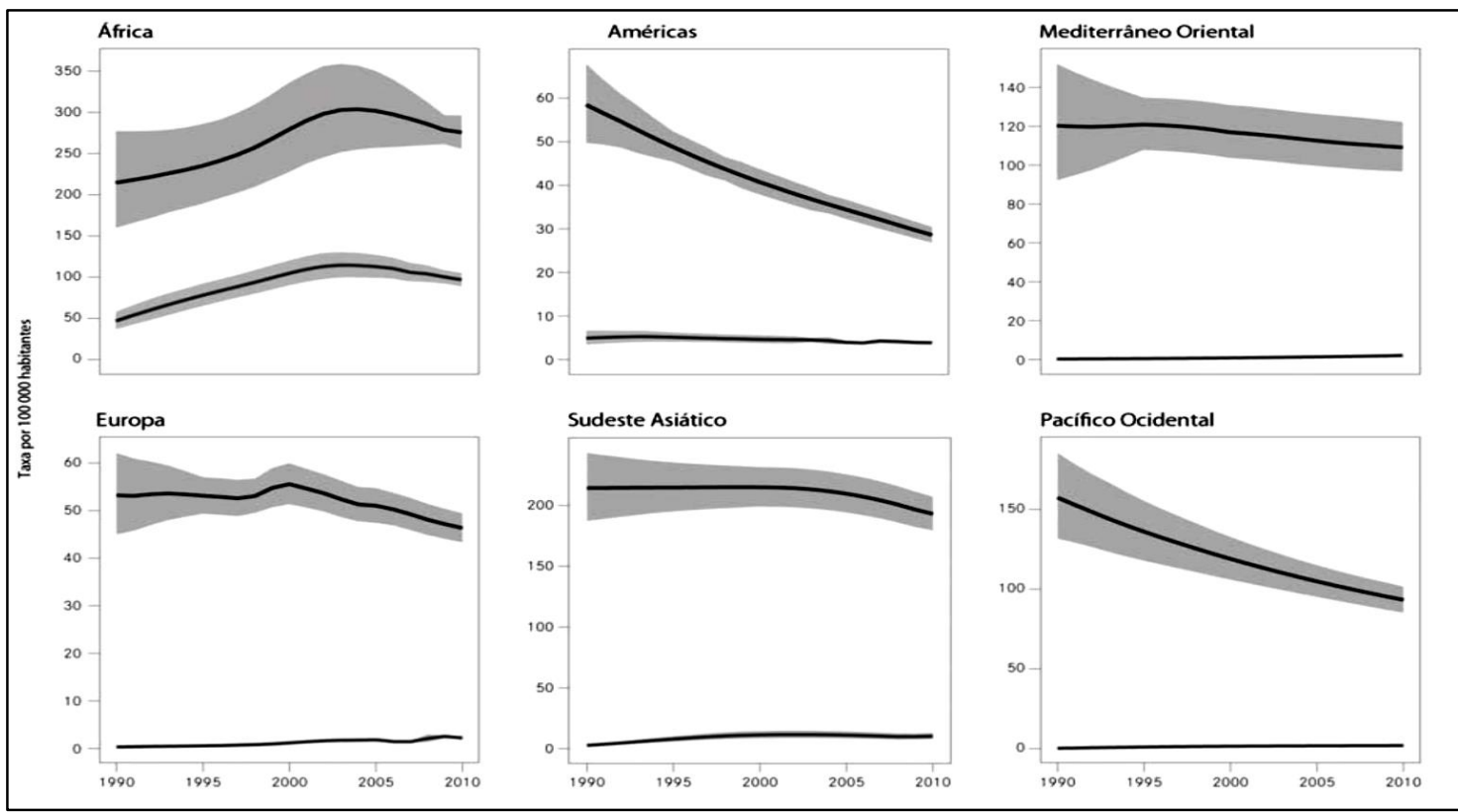

Figura 1 - Estimativa de taxa de incidência de tuberculose por região (1990-2010).

Fonte: Ministério da Saúde, (2015).

A linha superior indica a tendência estimada da taxa de incidência de tuberculose, a linha inferior indica a taxa estimada de incidência de coinfecção tuberculose/HIV. As áreas sombreadas representam margens de incerteza. [1].

São responsáveis por $81 \%$ de todos os casos de tuberculose no mundo 22 países, sendo os cinco primeiros a Índia (um quarto dos casos), China, África do Sul, Indonésia e Paquistão. O Brasil está em $17^{\circ}$ lugar nesse ranking [1].
Autoridades em saúde por todo o mundo tem se preocupado com a tuberculose. A Organização Mundial de Saúde declarou a tuberculose como emergência sanitária mundial em 1993. A iniciativa chamada Stop TB veio a seguir, estabelecendo metas para 2005 (detecção de $70 \%$ e cura de $85 \%$ dos casos), para 2015 e para 2050 (Tabela 1). Em 2000, a Organização das Nações Unidas estabeleceu as "Metas do Milênio", que incluíram o controle da tuberculose até 2015 [1].

Tabela 1 - Metas, alvos e indicadores para o controle de tuberculose [1].

Metas do Milênio Estipuladas para 2015

Meta 6: Combater o HIV/AIDS, malária e outras doenças.

Meta 6c: Para reverter a incidência da malária e de outras doenças importantes.

Indicador 6.9: Incidência, prevalência e taxas de mortalidade associadas à tuberculose.

Indicador 6.10: Proporção de casos de tuberculose detectados e curados dentro do tratamento diretamente supervisionado.

Parceria Stop TB estipula metas para 2015 e 2050

Até 2015: Reduzir a prevalência e taxa de mortalidade em 50\%, comparada com seus níveis em 1990.

Até 2050: Reduzir a incidência global de casos ativos de tuberculose para menos de 1 caso por 1 milhão de habitantes por ano.

Fonte: Ministério da Saúde, (2016).

A tuberculose é considerada uma prioridade do Ministério da Saúde do Brasil desde 2003 e uma das cinco doenças mais em foco atualmente. Está presente no programa Mais Saúde, na Programação das Ações de Vigilância em Saúde, no Pacto pela Vida, entre outros.

Está sendo discutida a inclusão da incidência de tuberculose como um indicador para o programa Brasil sem Miséria. O orçamento para o enfrentamento da tuberculose e 14 vezes maior daquele em 2002.
Estima-se que 57 milhões de pessoas estejam infectadas por M. tuberculosis no Brasil (Figura 2). Anualmente, são notificados 85 mil casos, sendo 71 mil casos novos, com uma incidência de 37,2/100.000 habitantes.

A tuberculose tem o dobro da incidência nos homens $(49,6 / 100.00$ habitantes) em relação as mulheres (24,6/100.000 habitantes). No Brasil, o grupo na faixa etária que vai dos 20 aos 49 anos e o mais atingido pela tuberculose, abrangendo em torno de $63 \%$ dos casos novos da doença registrados em 2009 [13]. 


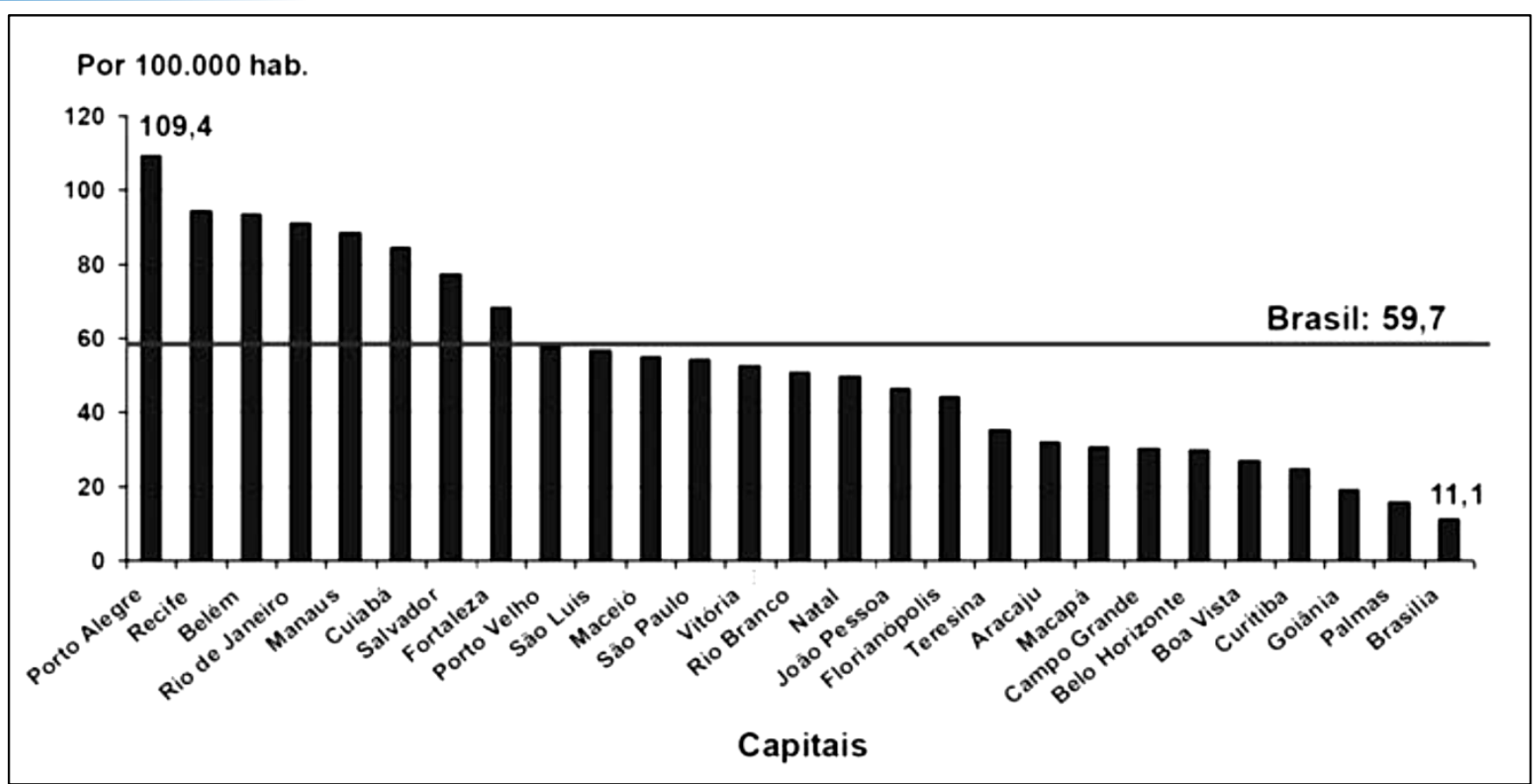

Figura 2 - Taxas de incidência nas capitais, Brasil, 2010.

Fonte: [13].

Nas populações mais vulneráveis, as taxas de incidência são maiores do que a média nacional da população geral. E duas vezes maior na população negra e quatro vezes maior na indígena. Na população carcerária, a taxa e 25 vezes maior e, entre os portadores de HIV, e 30 vezes maior. Na população vivendo em situação de rua, a taxa chega a ser 67 vezes maior [13][14][15][16].

Quanto ao risco anual de desenvolvimento de tuberculose ativa, a desnutrição e o diabetes elevam o risco em 2-4 vezes, o uso de imunossupressores eleva o risco em $2-12$ vezes, a silicose o aumenta em 8 - 34 vezes, e a infecção por HIV o aumenta em 50 100 vezes [13].

\section{II.1.3 TUBERCULOSE NO ESTADO DO PARÁ}

Metade dos casos de Tuberculose da Região Norte está no Pará e o estado ocupa o terceiro lugar no ranking nacional com maiores índices de novos casos da doença, segundo o Ministério da Saúde. Apesar do anúncio do Ministério de que os casos diminuíram, o panorama da doença local ainda é preocupante.

Segundo o [17], em 2011 foram 3.637 novos casos da doença e 79 mortes apenas no nosso território. Para se ter uma ideia, em todos os estados da Região, houve 7.282 casos. Se compararmos a proporção por habitante, o Pará ocupa a terceira colocação entre aqueles com maiores índices de novos casos da doença.

O [18] diz que, o levantamento também indica que, em dez anos, aumentou em 5\% os casos de tuberculose no Estado. Ao longo dessa década, foi observado movimento contrário na média nacional: decréscimo de cerca de $10 \%$. Em 2002, foram 3.278 novos casos no território paraense - 165 ocorrências a menos. Em compensação, 2012 indicou a primeira redução dos últimos cinco anos no Pará.

Em 2008, foram identificadas 3.340 pessoas infectadas; em 2009, 3.587; em 2010, 3.601; e no ano seguinte, 3.797. Na comparação entre 2011 e 2012, os novos casos paraenses de tuberculose caíram 10,2\%. No País, a redução de um ano para o outro, foi de 5,2\% - foram 73.748 em 2011.

Segundo [19], no Estado do Pará foi verificado para o ano de 2012 que a distribuição da Tuberculose não é homogênea, evidenciando a maioria dos casos em regiões próximas a região metropolitana da capital abrangendo $49,42 \%$ dos casos e, que a tendência de acometer pessoas de baixa escolaridade acompanha a tendência mundial.

\section{II.2 MÉTODO DAS MÉDIAS MÓVEIS}

Em tomada de decisão é bastante comum tratar problemas cujas decisões a serem tomadas são funções de fatos futuros. Assim, os dados descrevendo a situação de decisão precisam ser representativos do que ocorre no futuro. Em controle de estoques, por exemplo, as decisões são baseadas de acordo com a demanda para o item controlado durante um horizonte de planejamento específico. Em planejamento financeiro, faz-se necessário prever o padrão do fluxo de dinheiro em relação ao tempo [20].

\section{II.2.1 SÉRIES TEMPORAIS}

A maioria dos métodos de previsão estatística é baseada na utilização dos dados históricos a partir de uma série de tempo ou série temporal.

Uma série de tempo é uma série de observações de alguma quantidade de interesse (uma variável aleatória) em relação ao tempo. Assim, se $X_{i}$ é uma variável aleatória de interesse no tempo $\mathrm{i}$, e se observações são tomadas nos tempos $i=1,2, \ldots, t$, então os valores observados $\left\{X_{1}=x_{1}, X_{2}=x_{2}, \ldots, X_{t}=x_{t}\right\}$ são uma série de tempo [20]. 
Uma série temporal é uma descrição do passado, um procedimento lógico para realizar previsões é fazer uso desses dados históricos. Se os dados passados são indicativos do que se esperar no futuro, pode-se então postular um modelo matemático que é representativo do processo. $\mathrm{O}$ modelo pode então ser usado para gerar previsões [20].

Em situações reais, geralmente não se tem conhecimento da forma exata do modelo que gera a série temporal, com isso, fazse necessário escolher um modelo aproximado. Frequentemente, a escolha é feita observando os padrões de uma série temporal. Alguns padrões típicos são: a. Série de tempo é gerada por um processo com valor constante superposto a flutuações aleatórias;

b. Série de tempo é gerada por um processo linear superposto a flutuações aleatórias;

c. Série de tempo é gerada por um processo com valor constante superposto a variações sazonais e flutuações aleatórias.

A Figura 4, mostra exemplos gráficos dos padrões típicos $a, b$ e c.

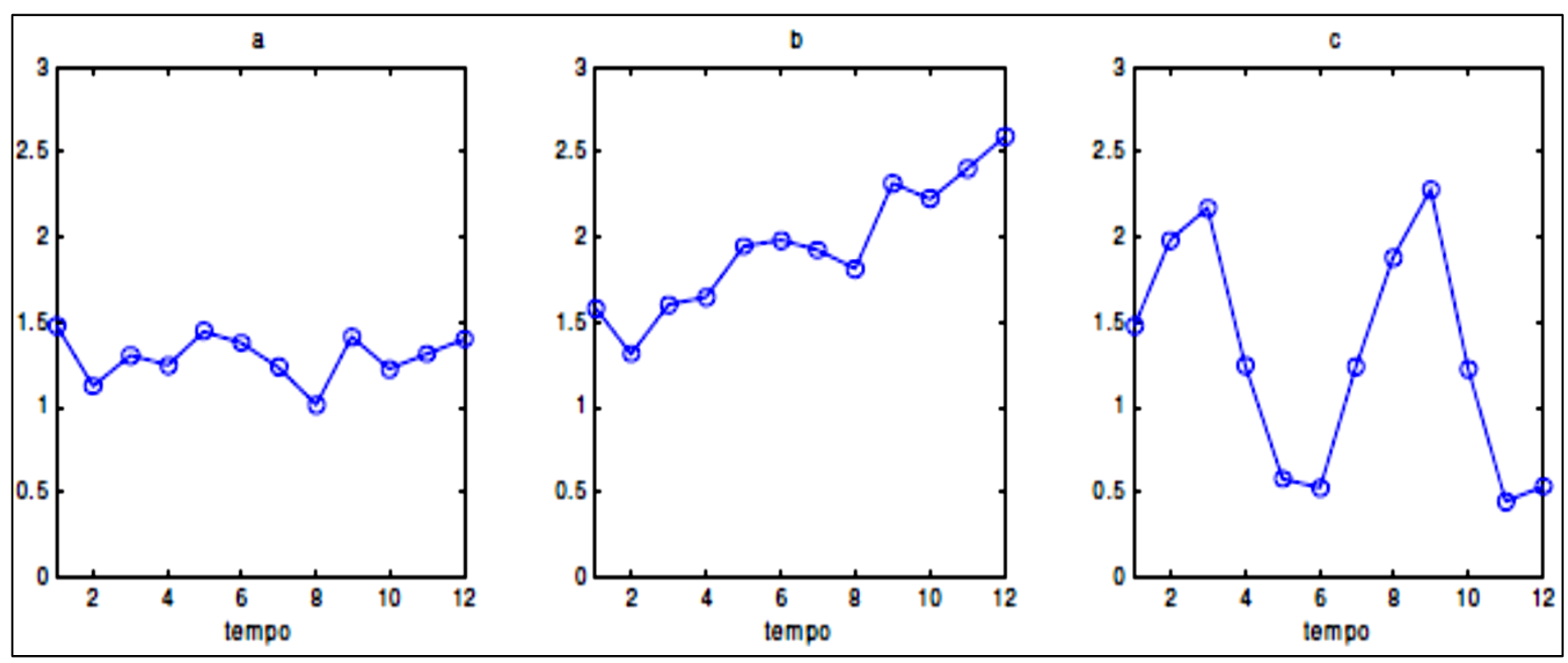

Figura 4 - Padrões típicos de séries temporais.

Fonte: Autores, (2015).

\section{II.2.2 MÉDIAS MÓVEIS SIMPLES (MMS)}

O método da média móvel simples é indicado para previsões de curto prazo onde as componentes de tendência e sazonalidade são inexistentes ou possam ser desprezadas (Figura 5) [21].

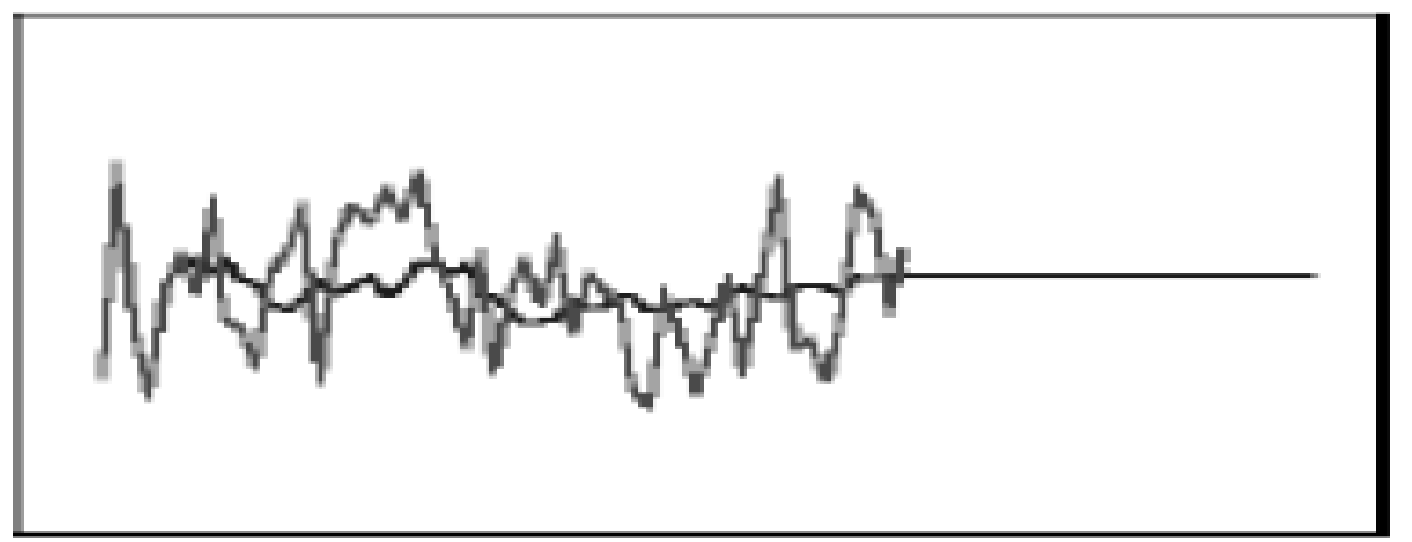

Figura 5 - Gráfico típico de dados de média móveis simples, mostrando o ajuste e a linha de previsão. Fonte: CB Predictor - User Manual - Selecionando métodos de previsão de séries temporais. P 3-10. 
Esse modelo nada mais é do que uma técnica simples de previsão exponencial onde são considerados os $\mathrm{k}$ últimos dados históricos e, com estes, é realizada uma média aritmética (ou ponderada) para prever o valor do próximo dado.

O número de observações $\mathrm{n}$ em cada cálculo da média, ou período, permanece constante e estipulado de maneira a tentar eliminar da melhor forma possível os componentes de tendência e sazonalidade [22][23[21].

Este método usa os $N$ últimos valores da série temporal $x_{t}$, como previsão para o tempo $t+1$. Portanto:

$$
\widehat{X}_{t+1}=\frac{1}{N} \sum_{i=t-N+1}^{t} x_{i}
$$

Onde:

$\widehat{X}_{t+1}=$ média móvel simples no final do período $t$;

$N=$ número de períodos incluídos em cada média;

$x_{i}=$ variável aleatória de interesse no tempo $i$.

Para saber se o modelo de séries temporais selecionado para realizar previsões é significativo estatisticamente deve-se quantificar o ajuste do modelo escolhido aos dados históricos existentes. Para isso, há diversas estatísticas:

\section{II.2.3 MEDIDAS DE ERROS NO PROCESSO DE PREVISÃO}

\section{II.2.3.1 MAD (Média dos Desvios Absolutos)}

Consiste na média da diferença (em módulo) entre os valores reais e preditos:

$$
M A D=\frac{1}{N} \sum_{t=1}^{n}\left|e_{t}\right|
$$

Onde:

$\mathrm{MAD}=$ média do desvio absoluto no final do período $t$;

$N=$ número de períodos incluídos em cada média;

$e_{t}=$ valor real menos o valor previsto ambos no tempo $t$.

Ela mede o erro absoluto e originalmente tornou-se muito popular (anteriormente às calculadoras) porque não exige cálculos de quadrados e raiz quadrada. Por enquanto é ainda muito confiável e amplamente utilizada, é muiţo acurada para dados distribuídos normalmente.

\section{II.2.3.2 MAPE (MÉDIA ARITMÉTICA DOS DESVIOS ABSOLUTOS EM PERCENTUAIS)} histórica:

Avalia a magnitude do erro com relação à série

$$
M A P E=\frac{1}{N} \sum_{t=1}^{n}\left|\frac{\varepsilon_{t}}{z_{t}} \times 100\right|
$$

Onde:

MAPE $=$ Média Aritmética dos Desvios Absolutos em Percentuais no final do período $t$;

$N=$ número de períodos incluídos em cada média;

$e_{t}=$ valor real menos a diferença do valor previsto ambos no tempo $t$;

$z_{t}=$ valor real no instante $t$.

É a mais popular das formas apresentadas para medir a acurácia da previsão [24]. Porém, o uso desta fórmula torna-se impossível quando a série temporal contém valores iguais à zero.

Por se tratar de erro relativo, ele não depende da escala, e com isso, permite comparar a acurácia da previsão entre séries de dados temporais de proporções diferentes.

\section{II.2.3.3 EQM (ERRO QUADRÁTICO MÉDIO)}

Destaca os grandes erros, comparados aos erros de menor magnitude:

$$
E Q M=\frac{1}{N} \sum_{t=1}^{n} e_{t}^{2}
$$

Onde:

$E Q M=$ Erro Quadrático Médio no final do período

$t$

$N$ = número de períodos incluídos em cada média;

$e_{t}^{2}=$ soma das diferenças entre valor real e o valor previsto, ponderadas pelo número de termos, ambos no tempo $t$.

\section{II.2.4 VANTAGENS E DESVANTAGENS DO MÉTODO}

As principais vantagens são [25]:

a) Simples aplicação;

b) É aplicável quando se tem um número pequeno de observações;

c) Permite uma flexibilidade grande devido à variação de $r$ de acordo com o padrão da série; e as desvantagens são [25]:

d) Deve ser utilizado somente para prever séries estacionárias, caso contrário a precisão das previsões obtidas será muito pequena, pois os pesos atribuídos às $r$ observações são todos iguais e nenhum peso é dado às observações anteriores à esse período;

e) Necessidade de armazenar pelo menos $(r-1)$ observações;

f) Dificuldade em determinar o valor de $r$. 
As desvantagens desse modelo estão relacionadas à falta de acurácia ao lidar com séries históricas que apresentam tendência ou sazonalidade já que, nesse método, a previsão para o próximo período envolve sempre a adição de novos dados e a desconsideração dos anteriores.

Uma alternativa para amenizar esse erro é a utilização da média ponderada para tentar construir um padrão mais próximo à realidade. A dificuldade na utilização da média móvel ponderada é a necessidade de conhecimento para se determinar os pesos a serem utilizados [26].

Na prática, o método das médias móveis não é utilizado frequentemente, pois o Método do Alisamento Exponencial Simples possui todas as vantagens anteriores e mais algumas que o tornam mais atraente [20].

Os modelos de alisamento exponencial podem ser de vários tipos: modelos de alisamento exponencial simples, modelos de alisamento exponencial duplo e modelos de Holt-Winters com e sem sazonalidade.

A diferença entre estes dois métodos é que, em se tratando do método de média móveis simples, as observações usadas para encontrar a previsão do valor futuro contribuem em igual proporção para o cálculo da previsão, enquanto que no método de alisamento exponencial simples cada observação da série recebe um peso diferente [25].

\section{MÉTODOS E PROCEDIMENTOS}

\section{III.1 COLETA DE DADOS}

Os dados do referido trabalho foram coletados a partir do banco de dados da Secretaria da Saúde do município de Capanema - PA, o qual oferece dados atualizados sobre diversas informações. No referido banco de dados, foi possível encontrar dados sobre o número de casos mensais, discriminado por sexo. $\mathrm{O}$ intervalo utilizado para estudo foi de JAN/2003 a DEZ/2014.

\section{III.2 ANÁLISE DOS DADOS}

Os dados coletados através da Secretaria de Saúde do município de Capanema - PA, expostos em tabela, foram tratados através do Método das Médias Móveis Simples, com a finalidade de se prever o número de casos de tuberculose para o ano de 2015. Para isso, foi utilizado o software EXCEL ${ }^{\circledR}$ para realizar as simulações e análises gráficas dos resultados. Em uma análise preliminar dos dados históricos, percebe-se que a incidência dos dados é de maior valor no período de maior incidência pluviométrica e maior umidade do ar.

\section{RESULTADOS E DISCUSSÕES}

\section{IV.1 PREVISÃO DOS CASOS DE TUBERCULOSE PARA O ANO DE 2015}

A Tabela 2 mostra os dados obtidos pela Secretaria de Saúde do município de Capanema - PA acerca dos casos de tuberculose totais no período de JAN/2003 a DEZ/2014, aos quais será feita a previsão para o período de Janeiro à Julho de 2015 pelo Método das Médias Móveis Simples.

Tabela 2 - Número de casos de Tuberculose totais no período de 2003 a 2014.

\begin{tabular}{c|c|c|c|c|c|c|c|c|c|c|c|c}
\hline Mês /Ano & $\mathbf{2 0 0 3}$ & $\mathbf{2 0 0 4}$ & $\mathbf{2 0 0 5}$ & $\mathbf{2 0 0 6}$ & $\mathbf{2 0 0 7}$ & $\mathbf{2 0 0 8}$ & $\mathbf{2 0 0 9}$ & $\mathbf{2 0 1 0}$ & $\mathbf{2 0 1 1}$ & $\mathbf{2 0 1 2}$ & $\mathbf{2 0 1 3}$ & $\mathbf{2 0 1 4}$ \\
\hline JAN & 7 & 4 & 6 & 1 & 3 & 4 & 0 & 0 & 1 & 2 & 7 & 4 \\
\hline FEV & 5 & 3 & 4 & 5 & 5 & 3 & 2 & 1 & 1 & 4 & 1 & 3 \\
\hline MAR & 1 & 1 & 7 & 3 & 6 & 2 & 3 & 4 & 0 & 7 & 0 & 4 \\
\hline ABR & 3 & 6 & 8 & 6 & 3 & 4 & 3 & 2 & 5 & 2 & 1 & 1 \\
\hline MAI & 2 & 4 & 3 & 7 & 2 & 2 & 3 & 2 & 1 & 0 & 1 & 2 \\
\hline JUN & 3 & 2 & 7 & 3 & 3 & 1 & 3 & 2 & 2 & 3 & 2 & 0 \\
\hline JUL & 6 & 2 & 6 & 4 & 6 & 7 & 3 & 3 & 3 & 1 & 7 & 4 \\
\hline AGO & 1 & 5 & 6 & 4 & 6 & 7 & 4 & 0 & 3 & 2 & 5 & 5 \\
\hline SET & 1 & 2 & 3 & 3 & 2 & 4 & 1 & 4 & 3 & 0 & 3 & 5 \\
\hline OUT & 5 & 4 & 3 & 0 & 4 & 3 & 3 & 2 & 5 & 2 & 7 & 0 \\
\hline NOV & 1 & 4 & 2 & 1 & 4 & 3 & 5 & 1 & 2 & 2 & 2 & 3 \\
\hline DEZ & 2 & 2 & 3 & 1 & 2 & 3 & 4 & 5 & 0 & 3 & 2 & 3 \\
\hline
\end{tabular}

Fonte: Autores, (2015). 
Bezerra, Silva \& Batista, ITEGAM-JETIA. Vol.02, № 08, pp.39-50. Dezembro, 2016.

A Tabela 3 mostra o resultado do ajuste pelo método das médias móveis simples dos dados acima expostos para os períodos $t=2, t=3, t=4, t=5, t=6, t=7$ e $t=8$.

\begin{tabular}{|c|c|c|c|c|c|c|c|c|c|c|}
\hline Ano & Mês & Período (t) & Casos (Zt) & $z(2)$ & $Z(3)$ & $Z(4)$ & $Z(5)$ & $Z(6)$ & $Z(7)$ & $Z(8)$ \\
\hline \multirow[t]{12}{*}{2003} & JAN & 1 & 7 & & & & & & & \\
\hline & FEV & 2 & 5 & & & & & & & \\
\hline & MAR & 3 & 1 & 6,000 & & & & & & \\
\hline & $A B R$ & 4 & 3 & 3,000 & 4,333 & & & & & \\
\hline & MAI & 5 & 2 & 2,000 & 3,000 & 4,000 & & & & \\
\hline & JUN & 6 & 3 & 2,500 & 2,000 & 2,750 & 3,600 & & & \\
\hline & JUL & 7 & 6 & 2,500 & 2,667 & 2,250 & 2,800 & 3,500 & & \\
\hline & AGO & 8 & 1 & 4,500 & 3,667 & 3,500 & 3,000 & 3,333 & 3,857 & \\
\hline & SET & 9 & 1 & 3,500 & 3,333 & 3,000 & 3,000 & 2,667 & 3,000 & 3,500 \\
\hline & OUT & 10 & 5 & 1,000 & 2,667 & 2,750 & 2,600 & 2,667 & 2,429 & 2,750 \\
\hline & NOV & 11 & 1 & 3,000 & 2,333 & 3,250 & 3,200 & 3,000 & 3,000 & 2,750 \\
\hline & DEZ & 12 & 2 & 3,000 & 2,333 & 2,000 & 2,800 & 2,833 & 2,714 & 2,750 \\
\hline \multirow[t]{12}{*}{2004} & JAN & 13 & 4 & 1,500 & 2,667 & 2,250 & 2,000 & 2,667 & 2,714 & 2,625 \\
\hline & FEV & 14 & 3 & 3,000 & 2,333 & 3,000 & 2,600 & 2,333 & 2,857 & 2,875 \\
\hline & MAR & 15 & 1 & 3,500 & 3,000 & 2,500 & 3,000 & 2,667 & 2,429 & 2,875 \\
\hline & $A B R$ & 16 & 6 & 2,000 & 2,667 & 2,500 & 2,200 & 2,667 & 2,429 & 2,250 \\
\hline & MAI & 17 & 4 & 3,500 & 3,333 & 3,500 & 3,200 & 2,833 & 3,143 & 2,875 \\
\hline & JUN & 18 & 2 & 5,000 & 3,667 & 3,500 & 3,600 & 3,333 & 3,000 & 3,250 \\
\hline & JUL & 19 & 2 & 3,000 & 4,000 & 3,250 & 3,200 & 3,333 & 3,143 & 2,875 \\
\hline & AGO & 20 & 5 & 2,000 & 2,667 & 3,500 & 3,000 & 3,000 & 3,143 & 3,000 \\
\hline & SET & 21 & 2 & 3,500 & 3,000 & 3,250 & 3,800 & 3,333 & 3,286 & 3,375 \\
\hline & OUT & 22 & 4 & 3,500 & 3,000 & 2,750 & 3,000 & 3,500 & 3,143 & 3,125 \\
\hline & NOV & 23 & 4 & 3,000 & 3,667 & 3,250 & 3,000 & 3,167 & 3,571 & 3,250 \\
\hline & DEZ & 24 & 2 & 4,000 & 3,333 & 3,750 & 3,400 & 3,167 & 3,286 & 3,625 \\
\hline \multirow[t]{12}{*}{2005} & JAN & 25 & 6 & 3,000 & 3,333 & 3,000 & 3,400 & 3,167 & 3,000 & 3,125 \\
\hline & FEV & 26 & 4 & 4,000 & 4,000 & 4,000 & 3,600 & 3,833 & 3,571 & 3,375 \\
\hline & MAR & 27 & 7 & 5,000 & 4,000 & 4,000 & 4,000 & 3,667 & 3,857 & 3,625 \\
\hline & $A B R$ & 28 & 8 & 5,500 & 5,667 & 4,750 & 4,600 & 4,500 & 4,143 & 4,250 \\
\hline & MAI & 29 & 3 & 7,500 & 6,333 & 6,250 & 5,400 & 5,167 & 5,000 & 4,625 \\
\hline & JUN & 30 & 7 & 5,500 & 6,000 & 5,500 & 5,600 & 5,000 & 4,857 & 4,750 \\
\hline & JUL & 31 & 6 & 5,000 & 6,000 & 6,250 & 5,800 & 5,833 & 5,286 & 5,125 \\
\hline & AGO & 32 & 6 & 6,500 & 5,333 & 6,000 & 6,200 & 5,833 & 5,857 & 5,375 \\
\hline & SET & 33 & 3 & 6,000 & 6,333 & 5,500 & 6,000 & 6,167 & 5,857 & 5,875 \\
\hline & OUT & 34 & 3 & 4,500 & 5,000 & 5,500 & 5,000 & 5,500 & 5,714 & 5,500 \\
\hline & NOV & 35 & 2 & 3,000 & 4,000 & 4,500 & 5,000 & 4,667 & 5,143 & 5,375 \\
\hline & DEZ & 36 & 3 & 2,500 & 2,667 & 3,500 & 4,000 & 4,500 & 4,286 & 4,750 \\
\hline \multirow[t]{3}{*}{2006} & JAN & 37 & 1 & 2,500 & 2,667 & 2,750 & 3,400 & 3,833 & 4,286 & 4,125 \\
\hline & FEV & 38 & 5 & 2,000 & 2,000 & 2,250 & 2,400 & 3,000 & 3,429 & 3,875 \\
\hline & MAR & 39 & 3 & 3,000 & 3,000 & 2,750 & 2,800 & 2,833 & 3,286 & 3,625 \\
\hline
\end{tabular}


Bezerra, Silva \& Batista, ITEGAM-JETIA. Vol.02, № 08, pp.39-50. Dezembro, 2016.

\begin{tabular}{|c|c|c|c|c|c|c|c|c|c|c|}
\hline & $A B R$ & 40 & 6 & 4,000 & 3,000 & 3,000 & 2,800 & 2,833 & 2,857 & 3,250 \\
\hline & MAI & 41 & 7 & 4,500 & 4,667 & 3,750 & 3,600 & 3,333 & 3,286 & 3,250 \\
\hline & JUN & 42 & 3 & 6,500 & 5,333 & 5,250 & 4,400 & 4,167 & 3,857 & 3,750 \\
\hline & JUL & 43 & 4 & 5,000 & 5,333 & 4,750 & 4,800 & 4,167 & 4,000 & 3,750 \\
\hline & AGO & 44 & 4 & 3,500 & 4,667 & 5,000 & 4,600 & 4,667 & 4,143 & 4,000 \\
\hline & SET & 45 & 3 & 4,000 & 3,667 & 4,500 & 4,800 & 4,500 & 4,571 & 4,125 \\
\hline & OUT & 46 & 0 & 3,500 & 3,667 & 3,500 & 4,200 & 4,500 & 4,286 & 4,375 \\
\hline & NOV & 47 & 1 & 1,500 & 2,333 & 2,750 & 2,800 & 3,500 & 3,857 & 3,750 \\
\hline & DEZ & 48 & 1 & 0,500 & 1,333 & 2,000 & 2,400 & 2,500 & 3,143 & 3,500 \\
\hline \multirow[t]{12}{*}{2007} & JAN & 49 & 3 & 1,000 & 0,667 & 1,250 & 1,800 & 2,167 & 2,286 & 2,875 \\
\hline & FEV & 50 & 5 & 2,000 & 1,667 & 1,250 & 1,600 & 2,000 & 2,286 & 2,375 \\
\hline & MAR & 51 & 6 & 4,000 & 3,000 & 2,500 & 2,000 & 2,167 & 2,429 & 2,625 \\
\hline & $A B R$ & 52 & 3 & 5,500 & 4,667 & 3,750 & 3,200 & 2,667 & 2,714 & 2,875 \\
\hline & MAI & 53 & 2 & 4,500 & 4,667 & 4,250 & 3,600 & 3,167 & 2,714 & 2,750 \\
\hline & JUN & 54 & 3 & 2,500 & 3,667 & 4,000 & 3,800 & 3,333 & 3,000 & 2,625 \\
\hline & JUL & 55 & 6 & 2,500 & 2,667 & 3,500 & 3,800 & 3,667 & 3,286 & 3,000 \\
\hline & AGO & 56 & 6 & 4,500 & 3,667 & 3,500 & 4,000 & 4,167 & 4,000 & 3,625 \\
\hline & SET & 57 & 2 & 6,000 & 5,000 & 4,250 & 4,000 & 4,333 & 4,429 & 4,250 \\
\hline & OUT & 58 & 4 & 4,000 & 4,667 & 4,250 & 3,800 & 3,667 & 4,000 & 4,125 \\
\hline & NOV & 59 & 4 & 3,000 & 4,000 & 4,500 & 4,200 & 3,833 & 3,714 & 4,000 \\
\hline & DEZ & 60 & 2 & 4,000 & 3,333 & 4,000 & 4,400 & 4,167 & 3,857 & 3,750 \\
\hline \multirow[t]{12}{*}{2008} & JAN & 61 & 4 & 3,000 & 3,333 & 3,000 & 3,600 & 4,000 & 3,857 & 3,625 \\
\hline & FEV & 62 & 3 & 3,000 & 3,333 & 3,500 & 3,200 & 3,667 & 4,000 & 3,875 \\
\hline & MAR & 63 & 2 & 3,500 & 3,000 & 3,250 & 3,400 & 3,167 & 3,571 & 3,875 \\
\hline & $A B R$ & 64 & 4 & 2,500 & 3,000 & 2,750 & 3,000 & 3,167 & 3,000 & 3,375 \\
\hline & MAI & 65 & 2 & 3,000 & 3,000 & 3,250 & 3,000 & 3,167 & 3,286 & 3,125 \\
\hline & JUN & 66 & 1 & 3,000 & 2,667 & 2,750 & 3,000 & 2,833 & 3,000 & 3,125 \\
\hline & JUL & 67 & 7 & 1,500 & 2,333 & 2,250 & 2,400 & 2,667 & 2,571 & 2,750 \\
\hline & AGO & 68 & 7 & 4,000 & 3,333 & 3,500 & 3,200 & 3,167 & 3,286 & 3,125 \\
\hline & SET & 69 & 4 & 7,000 & 5,000 & 4,250 & 4,200 & 3,833 & 3,714 & 3,750 \\
\hline & OUT & 70 & 3 & 5,500 & 6,000 & 4,750 & 4,200 & 4,167 & 3,857 & 3,750 \\
\hline & NOV & 71 & 3 & 3,500 & 4,667 & 5,250 & 4,400 & 4,000 & 4,000 & 3,750 \\
\hline & DEZ & 72 & 3 & 3,000 & 3,333 & 4,250 & 4,800 & 4,167 & 3,857 & 3,875 \\
\hline \multirow[t]{10}{*}{2009} & JAN & 73 & 0 & 3,000 & 3,000 & 3,250 & 4,000 & 4,500 & 4,000 & 3,750 \\
\hline & FEV & 74 & 2 & 1,500 & 2,000 & 2,250 & 2,600 & 3,333 & 3,857 & 3,500 \\
\hline & MAR & 75 & 3 & 1,000 & 1,667 & 2,000 & 2,200 & 2,500 & 3,143 & 3,625 \\
\hline & $A B R$ & 76 & 3 & 2,500 & 1,667 & 2,000 & 2,200 & 2,333 & 2,571 & 3,125 \\
\hline & MAI & 77 & 3 & 3,000 & 2,667 & 2,000 & 2,200 & 2,333 & 2,429 & 2,625 \\
\hline & JUN & 78 & 3 & 3,000 & 3,000 & 2,750 & 2,200 & 2,333 & 2,429 & 2,500 \\
\hline & JUL & 79 & 3 & 3,000 & 3,000 & 3,000 & 2,800 & 2,333 & 2,429 & 2,500 \\
\hline & AGO & 80 & 4 & 3,000 & 3,000 & 3,000 & 3,000 & 2,833 & 2,429 & 2,500 \\
\hline & SET & 81 & 1 & 3,500 & 3,333 & 3,250 & 3,200 & 3,167 & 3,000 & 2,625 \\
\hline & OUT & 82 & 3 & 2,500 & 2,667 & 2,750 & 2,800 & 2,833 & 2,857 & 2,750 \\
\hline
\end{tabular}


Bezerra, Silva \& Batista, ITEGAM-JETIA. Vol.02, № 08, pp.39-50. Dezembro, 2016.

\begin{tabular}{|c|c|c|c|c|c|c|c|c|c|c|}
\hline & NOV & 83 & 5 & 2,000 & 2,667 & 2,750 & 2,800 & 2,833 & 2,857 & 2,875 \\
\hline & DEZ & 84 & 4 & 4,000 & 3,000 & 3,250 & 3,200 & 3,167 & 3,143 & 3,125 \\
\hline \multirow[t]{12}{*}{2010} & JAN & 85 & 0 & 4,500 & 4,000 & 3,250 & 3,400 & 3,333 & 3,286 & 3,250 \\
\hline & FEV & 86 & 1 & 2,000 & 3,000 & 3,000 & 2,600 & 2,833 & 2,857 & 2,875 \\
\hline & MAR & 87 & 4 & 0,500 & 1,667 & 2,500 & 2,600 & 2,333 & 2,571 & 2,625 \\
\hline & $A B R$ & 88 & 2 & 2,500 & 1,667 & 2,250 & 2,800 & 2,833 & 2,571 & 2,750 \\
\hline & MAI & 89 & 2 & 3,000 & 2,333 & 1,750 & 2,200 & 2,667 & 2,714 & 2,500 \\
\hline & JUN & 90 & 2 & 2,000 & 2,667 & 2,250 & 1,800 & 2,167 & 2,571 & 2,625 \\
\hline & JUL & 91 & 3 & 2,000 & 2,000 & 2,500 & 2,200 & 1,833 & 2,143 & 2,500 \\
\hline & AGO & 92 & 0 & 2,500 & 2,333 & 2,250 & 2,600 & 2,333 & 2,000 & 2,250 \\
\hline & SET & 93 & 4 & 1,500 & 1,667 & 1,750 & 1,800 & 2,167 & 2,000 & 1,750 \\
\hline & OUT & 94 & 2 & 2,000 & 2,333 & 2,250 & 2,200 & 2,167 & 2,429 & 2,250 \\
\hline & NOV & 95 & 1 & 3,000 & 2,000 & 2,250 & 2,200 & 2,167 & 2,143 & 2,375 \\
\hline & DEZ & 96 & 5 & 1,500 & 2,333 & 1,750 & 2,000 & 2,000 & 2,000 & 2,000 \\
\hline \multirow[t]{12}{*}{2011} & JAN & 97 & 1 & 3,000 & 2,667 & 3,000 & 2,400 & 2,500 & 2,429 & 2,375 \\
\hline & FEV & 98 & 1 & 3,000 & 2,333 & 2,250 & 2,600 & 2,167 & 2,286 & 2,250 \\
\hline & MAR & 99 & 0 & 1,000 & 2,333 & 2,000 & 2,000 & 2,333 & 2,000 & 2,125 \\
\hline & $A B R$ & 100 & 5 & 0,500 & 0,667 & 1,750 & 1,600 & 1,667 & 2,000 & 1,750 \\
\hline & MAI & 101 & 1 & 2,500 & 2,000 & 1,750 & 2,400 & 2,167 & 2,143 & 2,375 \\
\hline & JUN & 102 & 2 & 3,000 & 2,000 & 1,750 & 1,600 & 2,167 & 2,000 & 2,000 \\
\hline & JUL & 103 & 3 & 1,500 & 2,667 & 2,000 & 1,800 & 1,667 & 2,143 & 2,000 \\
\hline & AGO & 104 & 3 & 2,500 & 2,000 & 2,750 & 2,200 & 2,000 & 1,857 & 2,250 \\
\hline & SET & 105 & 3 & 3,000 & 2,667 & 2,250 & 2,800 & 2,333 & 2,143 & 2,000 \\
\hline & OUT & 106 & 5 & 3,000 & 3,000 & 2,750 & 2,400 & 2,833 & 2,429 & 2,250 \\
\hline & NOV & 107 & 2 & 4,000 & 3,667 & 3,500 & 3,200 & 2,833 & 3,143 & 2,750 \\
\hline & DEZ & 108 & 0 & 3,500 & 3,333 & 3,250 & 3,200 & 3,000 & 2,714 & 3,000 \\
\hline \multirow[t]{12}{*}{2012} & JAN & 109 & 2 & 1,000 & 2,333 & 2,500 & 2,600 & 2,667 & 2,571 & 2,375 \\
\hline & FEV & 110 & 4 & 1,000 & 1,333 & 2,250 & 2,400 & 2,500 & 2,571 & 2,500 \\
\hline & MAR & 111 & 7 & 3,000 & 2,000 & 2,000 & 2,600 & 2,667 & 2,714 & 2,750 \\
\hline & $A B R$ & 112 & 2 & 5,500 & 4,333 & 3,250 & 3,000 & 3,333 & 3,286 & 3,250 \\
\hline & MAI & 113 & 0 & 4,500 & 4,333 & 3,750 & 3,000 & 2,833 & 3,143 & 3,125 \\
\hline & JUN & 114 & 3 & 1,000 & 3,000 & 3,250 & 3,000 & 2,500 & 2,429 & 2,750 \\
\hline & JUL & 115 & 1 & 1,500 & 1,667 & 3,000 & 3,200 & 3,000 & 2,571 & 2,500 \\
\hline & AGO & 116 & 2 & 2,000 & 1,333 & 1,500 & 2,600 & 2,833 & 2,714 & 2,375 \\
\hline & SET & 117 & 0 & 1,500 & 2,000 & 1,500 & 1,600 & 2,500 & 2,714 & 2,625 \\
\hline & OUT & 118 & 2 & 1,000 & 1,000 & 1,500 & 1,200 & 1,333 & 2,143 & 2,375 \\
\hline & NOV & 119 & 2 & 1,000 & 1,333 & 1,250 & 1,600 & 1,333 & 1,429 & 2,125 \\
\hline & DEZ & 120 & 3 & 2,000 & 1,333 & 1,500 & 1,400 & 1,667 & 1,429 & 1,500 \\
\hline \multirow[t]{4}{*}{2013} & JAN & 121 & 7 & 2,500 & 2,333 & 1,750 & 1,800 & 1,667 & 1,857 & 1,625 \\
\hline & FEV & 122 & 1 & 5,000 & 4,000 & 3,500 & 2,800 & 2,667 & 2,429 & 2,500 \\
\hline & MAR & 123 & 0 & 4,000 & 3,667 & 3,250 & 3,000 & 2,500 & 2,429 & 2,250 \\
\hline & $\mathrm{ABR}$ & 124 & 1 & 0,500 & 2,667 & 2,750 & 2,600 & 2,500 & 2,143 & 2,125 \\
\hline \multicolumn{11}{|c|}{48} \\
\hline
\end{tabular}


Bezerra, Silva \& Batista, ITEGAM-JETIA. Vol.02, № 08, pp.39-50. Dezembro, 2016.

\begin{tabular}{|c|c|c|c|c|c|c|c|c|c|c|}
\hline & MAI & 125 & 1 & 0,500 & 0,667 & 2,250 & 2,400 & 2,333 & 2,286 & 2,000 \\
\hline & JUN & 126 & 2 & 1,000 & 0,667 & 0,750 & 2,000 & 2,167 & 2,143 & 2,125 \\
\hline & JUL & 127 & 7 & 1,500 & 1,333 & 1,000 & 1,000 & 2,000 & 2,143 & 2,125 \\
\hline & AGO & 128 & 5 & 4,500 & 3,333 & 2,750 & 2,200 & 2,000 & 2,714 & 2,750 \\
\hline & SET & 129 & 3 & 6,000 & 4,667 & 3,750 & 3,200 & 2,667 & 2,429 & 3,000 \\
\hline & OUT & 130 & 7 & 4,000 & 5,000 & 4,250 & 3,600 & 3,167 & 2,714 & 2,500 \\
\hline & NOV & 131 & 2 & 5,000 & 5,000 & 5,500 & 4,800 & 4,167 & 3,714 & 3,250 \\
\hline & DEZ & 132 & 2 & 4,500 & 4,000 & 4,250 & 4,800 & 4,333 & 3,857 & 3,500 \\
\hline \multirow[t]{13}{*}{2014} & JAN & 133 & 4 & 2,000 & 3,667 & 3,500 & 3,800 & 4,333 & 4,000 & 3,625 \\
\hline & FEV & 134 & 3 & 3,000 & 2,667 & 3,750 & 3,600 & 3,833 & 4,286 & 4,000 \\
\hline & MAR & 135 & 4 & 3,500 & 3,000 & 2,750 & 3,600 & 3,500 & 3,714 & 4,125 \\
\hline & $A B R$ & 136 & 1 & 3,500 & 3,667 & 3,250 & 3,000 & 3,667 & 3,571 & 3,750 \\
\hline & MAI & 137 & 2 & 2,500 & 2,667 & 3,000 & 2,800 & 2,667 & 3,286 & 3,250 \\
\hline & JUN & 138 & 0 & 1,500 & 2,333 & 2,500 & 2,800 & 2,667 & 2,571 & 3,125 \\
\hline & JUL & 139 & 4 & 1,000 & 1,000 & 1,750 & 2,000 & 2,333 & 2,286 & 2,250 \\
\hline & AGO & 140 & 5 & 2,000 & 2,000 & 1,750 & 2,200 & 2,333 & 2,571 & 2,500 \\
\hline & SET & 141 & 5 & 4,500 & 3,000 & 2,750 & 2,400 & 2,667 & 2,714 & 2,875 \\
\hline & OUT & 142 & 0 & 5,000 & 4,667 & 3,500 & 3,200 & 2,833 & 3,000 & 3,000 \\
\hline & NOV & 143 & 3 & 2,500 & 3,333 & 3,500 & 2,800 & 2,667 & 2,429 & 2,625 \\
\hline & DEZ & 144 & 3 & 1,500 & 2,667 & 3,250 & 3,400 & 2,833 & 2,714 & 2,500 \\
\hline & & & EQM & 5,359 & 4,524 & 4,430 & 4,348 & 4,173 & 4,105 & 4,114 \\
\hline
\end{tabular}

Fonte: Autores, (2015).

A partir dos resultados obtidos na Tabela 3, foi gerado o gráfico com o ajuste dos dados sobre os casos de tuberculose no período de JAN/2003 a DEZ/2014. O gráfico da figura 6 mostra os resultados do ajuste dos dados coletados sobre os casos de tuberculose no município de Capanema/PA.

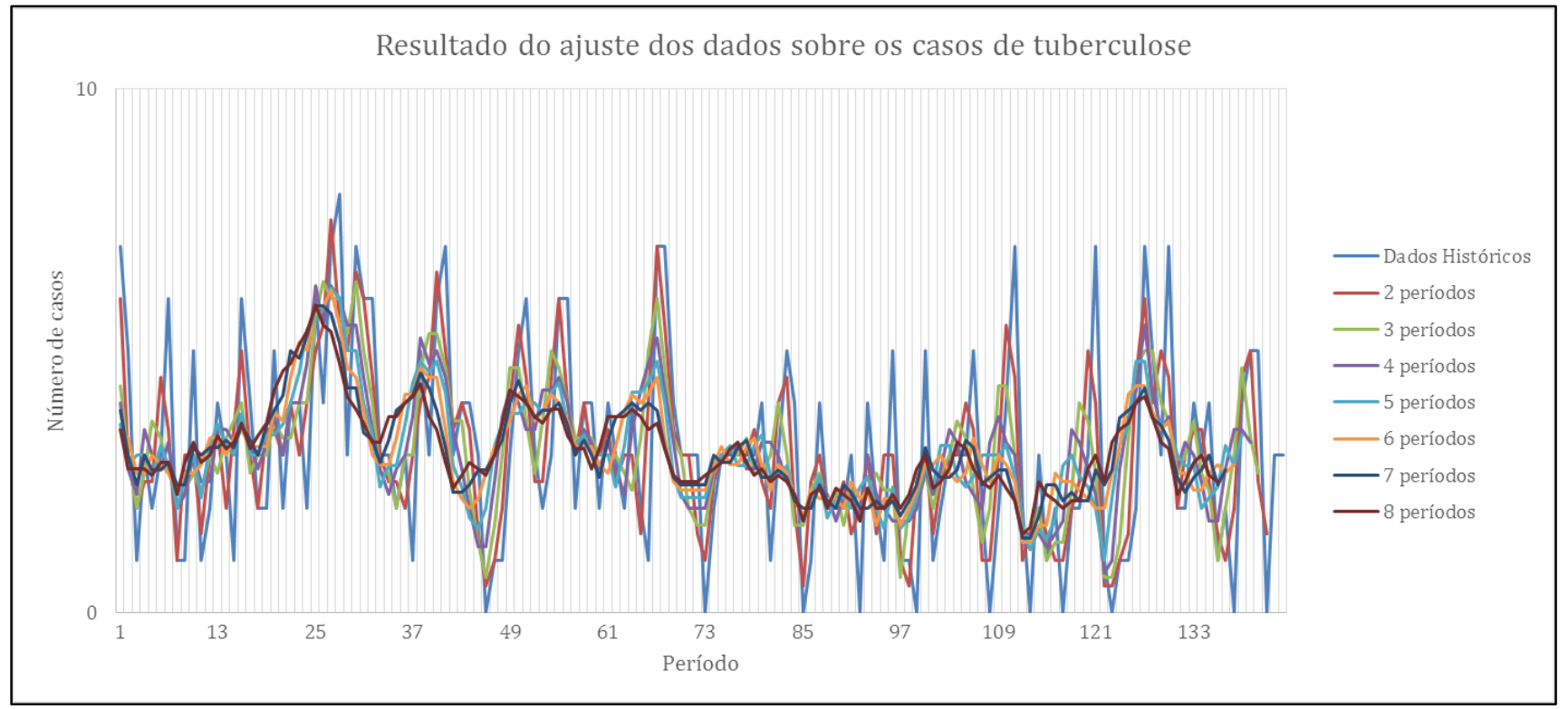

Figura 6 - Gráfico do resultado do ajuste dos dados sobre os casos de tuberculose no período de JAN/2003 a DEZ/2014. Fonte: Os Autores (2015). 
Analisando os resultados obtidos pela Tabela 3 e o gráfico, percebe-se que a média móvel simples de 7 períodos foi a que melhor representou os dados sobre os casos de tuberculose entre os anos de 2003 a 2014, pois o seu erro quadrático médio foi o menor obtido dentre todos os períodos testados. Desta forma, utilizando a média móvel simples de 7 períodos (MMS7P), foi possível prever o número de casos de tuberculose para o período de janeiro a julho do ano de 2015 e, seus resultados estão expostos na Tabela 4.

Tabela 4 - Resultado da previsão do número de casos de tuberculose para o ano de 2015 para os meses de janeiro a julho.

\begin{tabular}{c|c|c|c}
\hline Ano & Mês & Período (t) & $\mathbf{Z ~ ( 7 ) ~}$ \\
\hline \multirow{4}{*}{015} & JAN & 1 & 2,857 \\
\cline { 2 - 4 } & FEV & 2 & 3,333 \\
\cline { 2 - 4 } & MAR & 3 & 3,200 \\
\cline { 2 - 4 } & ABR & 4 & 2,750 \\
\cline { 2 - 4 } & MAI & 5 & 2,000 \\
\cline { 2 - 4 } & JUN & 6 & 3,000 \\
\cline { 2 - 4 } & JUL & 7 & 3,000 \\
\hline
\end{tabular}

Fonte: Os Autores, (2015).

Desta forma, como apresentado na Tabela 4, são previstos aproximadamente 20 casos de tuberculose para o ano de 2015 no período de Janeiro a Julho no município de Capanema/PA.

\section{CONCLUSÃO}

A tuberculose (TB) é uma doença infectocontagiosa e, quando diagnosticada o quanto antes, maior é a sua chance de cura. A incidência de casos de TB vem diminuindo ao passar dos anos devido aos investimentos dos governos no tratamento da doença. Dados estatísticos de previsão de dados de como a doença vem atingindo a população ao longo dos anos são de grande importância para que se possa prever recursos para o diagnóstico e/ou tratamento desta.

O método das médias móveis simples mostra-se como uma importante ferramenta quando os dados se apresentam em grande aleatoriedade, sem períodos de sazonalidade.

Portanto, o resultado obtido para os meses de janeiro a julho de 2015, foi de 20 casos de tuberculose no município de Capanema/PA.

Percebe-se que esse resultado foi coerente, devido a comparação com os anos anteriores registrados no município.

\section{AGRADECIMENTOS}

Ao Instituto de Tecnologia Galileo da Amazônia (ITEGAM) e ao PPGEP da UFPA pelo apoio a esta pesquisa.

\section{REFERÊNCIAS}

[1] World Health Organization - Global Tuberculosis Control $-2011$.

[2] RUFFINO-NETO, A. Tuberculose: a calamidade negligenciada. Revista da Sociedade Brasileira de Medicina Tropical, 35(1): 51-58, jan-fev, 2002.

[3] NATAL, S. Tuberculose na criança. Boletim de Pneumologia Sanitária. 8: 21-25, 2000.

[4] AMARAnTE, J.M.; COSTA, V. L. A. A tuberculose nas comunidades indígenas brasileiras na virada do século. Boletim de Pneumologia Sanitária 8: 5-12, 2000.
[5] BARUZZI, R.G.; BARROS, V.L.; RODRIGUES, D.; SOUZA, A. L. M.; PAGLIARO, H. Saúde e doença em índios Panará (Kreen-Akarôe) após vinte e cinco anos de contato com o nosso mundo, com ênfase na ocorrência de tuberculose (Brasil Central). Cadernos de Saúde Pública 17: 407-412, 2001.

[6] Marques AMC, Cunha RV. A medicação assistida e os índices de cura de tuberculose e de abandono de tratamento na população indígena Guarani-Kaiwá no Município de Dourados, Mato Grosso do Sul, Brasil. Cad Saúde Pública 2003; 19(5):140511

[7] SANTOS, R. V., COIMBRA JR, C. E. A. Cenários e tendências da saúde e da epidemiologia dos povos indígenas no Brasil. In: Coimbra Jr CEA, Santos RV, Escobar AL (orgs) Epidemiologia e saúde dos povos indígenas no Brasil, Editora FIOCRUZ, Rio de Janeiro, p.13-48, 2003

[8] GARNELO, L, MACEDO, G, BRANDÃO, L. C. Os povos indígenas e a construção das políticas de saúde no Brasil. Organização Pan-Americana de Saúde, Brasília - DF, 2003.

[9] ESCOBAR, A. L., COIMBRA JR, C. E. A., CAMACHO LA, PORTELA, M. C. Tuberculose em populações indígenas de Rondônia, Amazônia, Brasil. Cadernos de Saúde Pública 17: 285298, 2001.

[10] HARAKI, A. Y. Tuberculose - A Peste Cinzenta: a importância a pasteurização do leite. In: Boletim Informativo Via-Láctea. Ano 01. №8. Dezembro/2009.

[11] BUENO, E. \& TAITELBAUM, P. Vendendo Saúde: a história. Agência Nacional de Vigilância Sanitária, 2008. Disponível em: www.anvisa.gov.br/vendendo+saude. Acesso em:

10 de maio de 2014.

[12] Adorno, Sergio. Exclusão socioeconômica e violência urbana. Sociologias. Porto Alegre, ano 4, n. 8, p. 84-135, jul.-dez. 2002. 